\title{
Benzo-Fused Periacenes or Double Helicenes? Different Cyclodehydrogenation Pathways on Surface and in Solution
}

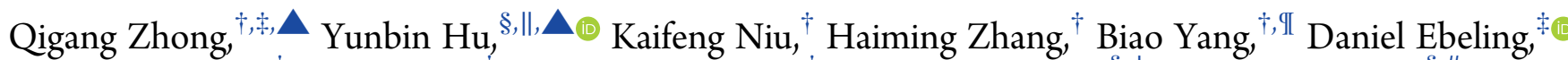

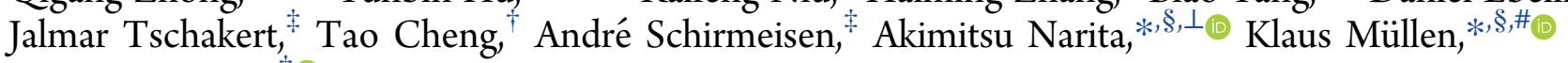 \\ and Lifeng Chi* ${ }^{\dagger}$ (1)
}
${ }^{\dagger}$ Institute of Functional Nano \& Soft Materials (FUNSOM), Jiangsu Key Laboratory for Carbon-Based Functional Materials and Devices, Joint International Research Laboratory of Carbon-Based Functional Materials and Devices, Soochow University, Suzhou 215123, China
${ }^{\ddagger}$ Institute of Applied Physics, Justus-Liebig University, Heinrich-Buff-Ring 16, 35392 Giessen, Germany
${ }^{\S}$ Max Planck Institute for Polymer Research, 55128 Mainz, Germany
"Department of Organic and Polymer Chemistry, College of Chemistry and Chemical Engineering, Central South University, Changsha, Hunan 410083, China
${ }^{\perp}$ Organic and Carbon Nanomaterials Unit, Okinawa Institute of Science and Technology Graduate University, Okinawa 904-0495, Japan
\#Institute of Physical Chemistry, Johannes Gutenberg University Mainz, Duesbergweg 10-14, 55128 Mainz, Germany

\section{Supporting Information}

\begin{abstract}
Controlling the regioselectivity of $\mathrm{C}-\mathrm{H}$ activation in unimolecular reactions is of great significance for the rational synthesis of functional graphene nanostructures, which are called nanographenes. Here, we demonstrate that the adsorption of tetranaphthyl- $p$-terphenyl precursors on metal surfaces can completely change the cyclodehydrogenation route and lead to obtaining planar benzo-fused perihexacenes rather than double [7]helicenes during solution synthesis. The course of the on-surface planarization reactions is monitored using scanning probe microscopy, which unambiguously reveals the formation of dibenzoperihexacenes and the structures of reaction intermediates. The regioselective planarization can be attributed to the flattened adsorption geometries and the reduced flexibility of the precursors on the surfaces, in addition to the different mechanism of

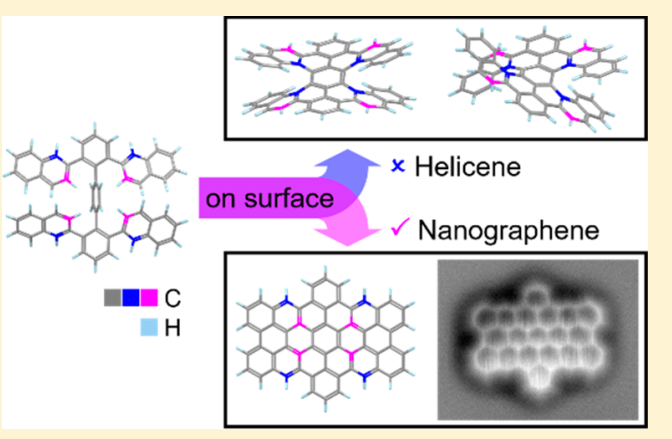
the on-surface cyclodehydrogenation from that of the solution counterpart. We have further achieved the on-surface synthesis of dibenzoperioctacene by employing a tetra-anthryl-p-terphenyl precursor. The energy gaps of the new nanographenes are measured to be approximately $2.1 \mathrm{eV}$ (dibenzoperihexacene) and $1.3 \mathrm{eV}$ (dibenzoperioctacene) on a Au(111) surface. Our findings shed new light on the regioselectivity in cyclodehydrogenation reactions, which will be important for exploring the synthesis of unprecedented nanographenes.
\end{abstract}

\section{INTRODUCTION}

Nanographenes (NGs) are regarded as segments of graphene featuring nanometer sizes in one or two dimensions. ${ }^{1}$ The size control, especially at the sub-10 nm scale, has been proven to induce a quantum confinement effect and allow for the tuning of the electronic band gap. ${ }^{2,3}$ The unique, structure-dependent properties of NGs qualify them for various applications in molecular electronics, ${ }^{4}$ spintronics, ${ }^{5}$ and optoelectronics. ${ }^{6}$ Since the electronic properties of NGs sensitively depend on their size ${ }^{7}$ and edge geometries, ${ }^{8}$ it is mandatory to fabricate NGs with atomic precision. To this end, bottom-up molecular synthesis is the most suitable approach for providing NG molecules, i.e., large polycyclic aromatic hydrocarbons (PAHs) with uniform and defined chemical structures. The synthesis of NG molecules has typically been carried out through the oxidative cyclodehydrogenation of tailor-made oligoarylene precursors in solution in the presence of Lewis acids and oxidants. $^{9-19}$ Nevertheless, the cyclodehydrogenation in solution is sometimes hampered by side reactions furnishing undesired and often inseparable mixtures of products. ${ }^{20-24} \mathrm{On}$ the other hand, cyclodehydrogenation on metal surfaces has emerged during the past decade as an alternative and complementary method enabling the synthesis of atomically precise NG molecules under ultrahigh vacuum (UHV) conditions. $^{25-27}$ The on-surface cyclodehydrogenation proceeds at elevated temperatures, typically at $>400{ }^{\circ} \mathrm{C}$, with the help of the catalytic effect of the metal surfaces such as

Received: February 1, 2019

Published: April 16, 2019 
$\mathrm{Au}(111), \mathrm{Ag}(111)$, and $\mathrm{Cu}(111)$. This process is most likely accompanied by the release of hydrogen, ${ }^{28}$ which is in contrast to the solution reaction that is regarded to include cationic intermediates with the elimination of protons; however, the mechanisms of both cases are not yet fully understood. Onsurface synthesis allowed for the formation of unstable PAHs, in particular, higher acenes, which could not be obtained in solution. ${ }^{29,30}$ It is essential, therefore, that the resulting product structures can be visualized at an atomic resolution using in situ scanning probe microscopy (SPM). ${ }^{25,26,31-34}$ However, little is known about the influence of metal surfaces on the selectivity of the cyclodehydrogenation reaction, especially when different isomers can be envisaged.

Periacenes, which are zigzag-edge NG molecules with two rows of peri-fused linear acenes, are highly attractive due to their intriguing properties, such as low energy gaps and openshell biradical character. ${ }^{35,36}$ In addition to the smallest periacenes, perylene, and bisanthene, ${ }^{37-43}$ the synthesis of peritetracene with bulky substituents was recently achieved in solution. ${ }^{44,45}$ The synthesis of peripentacene was demonstrated on a $\mathrm{Au}(111)$ surface under UHV to be the largest pristine periacene reported to date. ${ }^{33}$ On the other hand, we have synthesized OBO-doped perihexacene on a $\mathrm{Au}(111)$ surface, ${ }^{46}$ which was hitherto the largest periacene analogue in the literature. However, its energy gap was relatively large and the synthesis of a pure hydrocarbon structure of perihexacene has remained challenging. Recently, we attempted the synthesis of dibenzoperihexacene $3 a$ as a hydrocarbon analogue of perihexacene. Remarkably enough, the oxidative cyclodehydrogenation of the tetranaphthyl-p-terphenyl precursor 1a in solution selectively provided the benzo-fused double [7]helicene $2 a{ }^{47}$ thus leaving the synthesis of dibenzoperihexacene elusive (Figure 1).

Herein, we investigated the on-surface cyclodehydrogenation of tetranaphthyl-p-terphenyl precursors $\mathbf{1 a}$ and $\mathbf{1} \mathbf{b}$ on $\mathrm{Cu}(110)$ and $\mathrm{Au}(111)$ surfaces $(\mathrm{Cu}(111)$ was also used for comparison), which led to the formation of dibenzoperihexacenes with distinct regioselectivity of the reaction compared with the solution synthesis. We have further achieved the onsurface synthesis of dibenzoperioctacene, which is the higher homologue of dibenzoperihexacene, from the tetra-anthryl- $p$ terphenyl precursor 5. Regarding the reaction mechanisms, partially fused intermediate structures could also be elucidated by conducting on-surface reactions at different temperatures while monitoring the progress using scanning tunneling microscopy (STM) and bond-resolution atomic force microscopy (AFM) ${ }^{48}$ images. The pristine and intermediate structures along the reaction combined with density functional theory (DFT) calculations provided insights into the planarization. Scanning tunneling spectroscopy (STS; $\mathrm{d} I / \mathrm{d} V$ vs $V$ ) revealed that their energy gaps were approximately 2.1 $\mathrm{eV}$ (dibenzoperihexacene) and $1.3 \mathrm{eV}$ (dibenzoperioctacene), thereby demonstrating the significant decrease of the gap upon the extension of the NG core. To the best of our knowledge, the dibenzoperihexacene and dibenzoperioctacene that are reported here are to date the largest hydrocarbon periacene analogues.

\section{RESULTS AND DISCUSSION}

First, we deposited a submonolayer of $4,4^{\prime \prime}$-di-tert-butyl2,2",6,6"-tetra(2-naphthyl)-p-terphenyl (TNTP-tBu, 1a) onto a room temperature $\mathrm{Cu}(110)$ surface using molecular sublimation at $620 \mathrm{~K}$. As seen in Figure 2a, the molecules

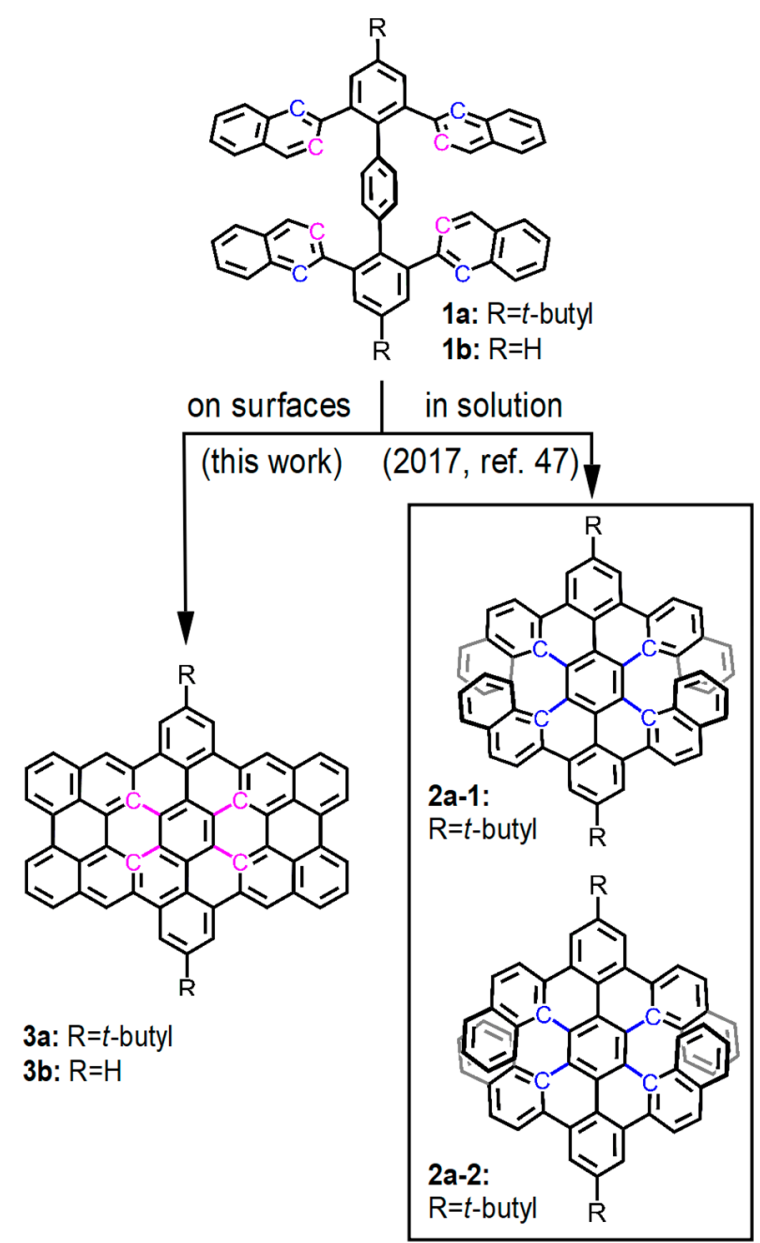

Figure 1. Chemical structures of the tetranaphthyl-p-terphenyl precursor $\mathbf{1}$ (TNTP-tBu $\mathbf{1 a}$ and TNTP $\mathbf{1 b}$ ) and its different cyclodehydrogenation products, the double [7] carbohelicenes 2a-1 and $\mathbf{2 a - 2}$ that were synthesized in solution and the dibenzoperihexacene 3 formed on metal surfaces in this work.

were scattered on the $\mathrm{Cu}(110)$ surfaces without forming a regular assembly. Most of the molecules of 1a (with the axis across the two tert-butyl ( $t \mathrm{Bu})$ groups) were adsorbed parallel to the $[1-10]$ direction of $\mathrm{Cu}(110)$. As shown in Figure 2d, g, and $j$ and Figure S1, the four naphthyl branches were arranged nearly parallel to each other. This configuration is advantageous for the $\mathrm{C}-\mathrm{C}$ bond formation at the $\beta$-position of the naphthyl group (highlighted in pink in Figure 1) because the reaction at the $\alpha$-position (highlighted in blue in Figure 1) would involve an extra flipping process of the naphthyl group. Two naphthyl groups (the upper two in Figure 2d and g) were relatively flat, making them invisible to AFM imaging with a constant height at the tip height $+50 \mathrm{pm}$ (Figure $2 \mathrm{~g}$ ) and a constant-current mode (Figure $\mathrm{S} 1 \mathrm{~b}$ and $\mathrm{c}$ ). In contrast, the other two naphthyl groups and the central benzene ring were tilted with respect to the surface plane due to intramolecular steric hindrance. This configuration of adsorbed TNTP- $t \mathrm{Bu}$ molecules was verified by the DFT calculated structures (Figures $3 \mathrm{a}$ and $\mathrm{b}$ ) and the simulated AFM image (Figure 3c). Interestingly, a $90^{\circ}$ rotation of the molecules was observed after annealing at $460 \mathrm{~K}$ for $30 \mathrm{~min}$, but pristine TNTP- $t \mathrm{Bu}$ was still predominant (Figure S2b and Figure S3a-d).

To our delight, $\mathrm{C}-\mathrm{C}$ bond formation at the $\beta$-positions of the naphthyl groups was observed when annealing the sample 


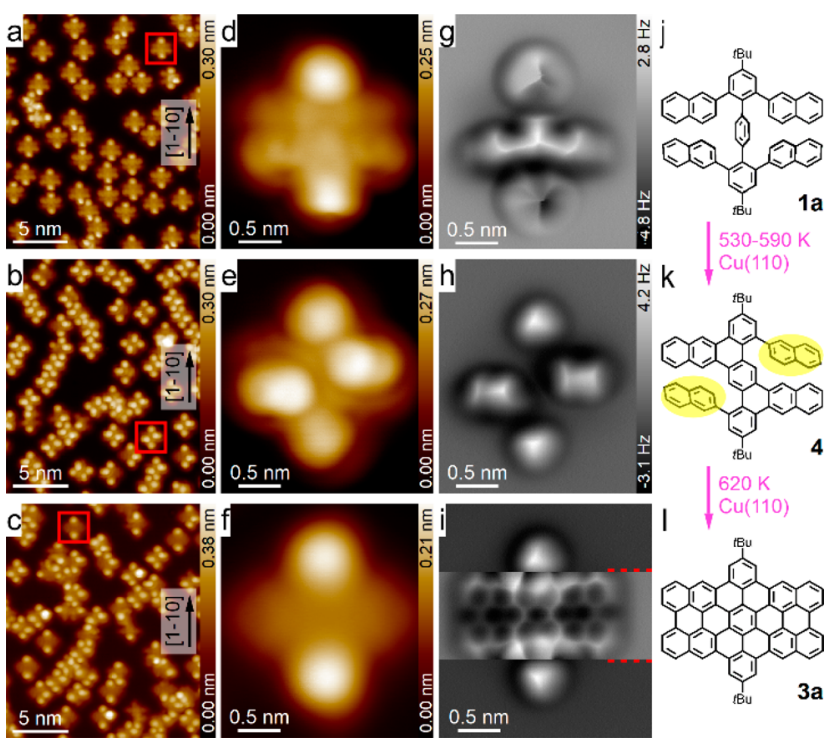

Figure 2. Cyclodehydrogenation reactions of TNTP-tBu 1a on $\mathrm{Cu}(110)$. (a-c) STM overview images that were obtained after the evaporation of 1a (a), and after annealing the sample at $590 \mathrm{~K}$ (b) and at $620 \mathrm{~K}(\mathrm{c}) .(\mathrm{d}-\mathrm{f})$ STM zoomed-in images of an initial TNTP$t \mathrm{Bu}$ molecule, a typical intermediate molecule and a final product molecule, respectively. ( $g-i)$ Constant-height frequency shift AFM images of the three molecules shown in $(d-f) ;(j-k)$ corresponding chemical structures. Imaging parameters: $(\mathrm{a}-\mathrm{e}) 0.1 \mathrm{~V}, 10 \mathrm{pA}$; (f) 10 $\mathrm{mV}, 100 \mathrm{pA}$; and tip heights $Z=+50 \mathrm{pm}(\mathrm{g}),+130 \mathrm{pm} \mathrm{(h)}$, and +120 pm (i) $(Z=-120 \mathrm{pm}$ for the region between the two red dashed lines in (i)), where the \pm signs represent the increase/decrease of the tipsample distance relative to the STM set point $(0.1 \mathrm{~V}, 10 \mathrm{pA})$ on the adjacent $\mathrm{Cu}(110)$ surfaces. Since the contrast in (i) is separately optimized for the middle section and the top/bottom sections, an overall color bar is not given.

at $530 \mathrm{~K}$ or higher, thereby leading to the formation of dibenzoperihexacene $\mathbf{3 a}$ instead of double [7]helicene 2a, as previously obtained in the solution synthesis (Figure 2; see Figure S2 for the whole annealing processes). Between 530 and $590 \mathrm{~K}$, intermediates such as 4 , resulting from the incomplete cyclodehydrogenation, were detected with different adsorption geometries and orientations. The chemical structures of the intermediates were confirmed by high-resolution AFM images and computer simulations (Figures $3 \mathrm{e}-\mathrm{h}$ and $\mathrm{S} 3 \mathrm{e}-\mathrm{h}$ ). Intermediate 4 was more distorted than the pristine precursor 1a, thus leaving two of the four naphthyl groups appearing as bright lobes in the STM image (Figure 2e); they are highlighted by the two yellow ovals in Figure $2 k$, while the other two naphthyl groups are fused to the central benzene ring. At $590 \mathrm{~K}$, molecules with only one remaining naphthyl group were also found, e.g., in Figure S3i-l. Nevertheless, no other intermediate structure was found, indicating that the planarization of each naphthyl group from structure 4 was accompanied by subsequent or simultaneous formation of three $\mathrm{C}-\mathrm{C}$ bonds. Elevating the annealing temperature to 620 $\mathrm{K}$ induced the complete planarization of some molecules $(\sim 10 \%(5 / 52)$, as seen in Figure $2 c, f, i$, and 1$)$. After annealing at $640 \mathrm{~K}$, most of the molecules have been planarized. Some of the molecules were connected with each other due to the removal of the tert-butyl groups followed by intermolecular C$\mathrm{C}$ bond formation (Figure S2f). It should be noted that the dibenzoperihexacene structure of $\mathbf{3 a}$ is not completely flat because of the bulkiness of the $t \mathrm{Bu}$ groups, which makes the

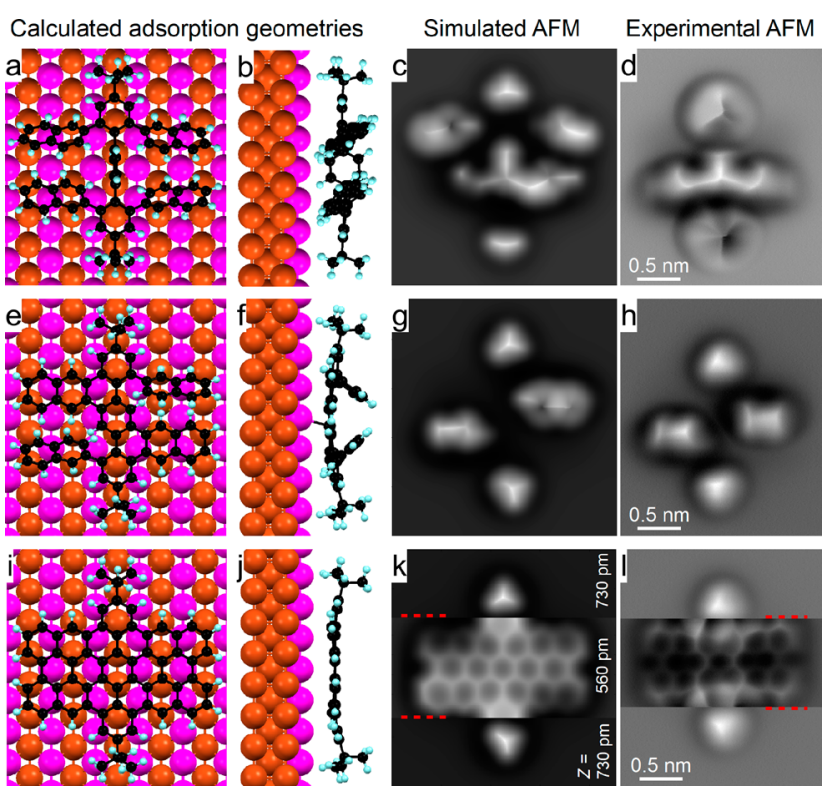

Figure 3. Calculated adsorption geometries and simulated AFM images of the initial 1a, intermediate 4 , and final product $3 \mathbf{a}$ of TNTP- $t \mathrm{Bu}$ on $\mathrm{Cu}(110)$. (a, b, e, f, i, j) Top and side views of the calculated adsorption geometries of an initial molecule $(a, b)$, an intermediate (e, f), and a final product molecule $(i, j)$ on $\mathrm{Cu}(110)$. The $\mathrm{C}, \mathrm{H}$, first layer $\mathrm{Cu}$, and bulk $\mathrm{Cu}$ atoms are represented by the black, blue, violet, and red spheres, respectively. (c, g, k) Simulated constant-height AFM images of an initial molecule (c), an intermediate $(\mathrm{g})$, and a final product molecule $(\mathrm{k})$ adsorbed on $\mathrm{Cu}(110)$. (d, h, l) Experimental constant-height AFM images of an initial molecule (d), an intermediate $(\mathrm{h})$, and a final product molecule (l) adsorbed on $\mathrm{Cu}(110)$. Imaging parameters: images (d, h, and l) are the same as Figure $2 \mathrm{~g}, \mathrm{~h}$, and $\mathrm{i}$.

middle part of the molecule higher while the two sides are bent down due to the strong interaction with the substrate, as shown in Figure 2i and Figure $3 \mathrm{j}$ and $\mathrm{k}$.

As mentioned above, precursor 1a was distributed separately on $\mathrm{Cu}(110)$ surfaces, thus implying that there is a stronger molecule-surface interaction than molecule-molecule interaction. To exclude possible special template and/or catalytic effects of $\mathrm{Cu}(110)$ as the driving force for the planarization, control experiments on $\mathrm{Cu}(111)$ and $\mathrm{Au}(111)$ were conducted. Unlike the scattered distribution on $\mathrm{Cu}(110)$, precursor 1a initially assembled into a two-dimensional monolayer on $\mathrm{Cu}(111)$ and $\mathrm{Au}(111)$ (see Figure $\mathrm{S} 4 \mathrm{c}$ and d). The molecules were adsorbed in a more twisted shape presumably because of the interplay of a weaker moleculesurface interaction on $\mathrm{Cu}(111) / \mathrm{Au}(111)$ and the intramolecular geometric relaxation. The same planarized products were observed when annealing at approximately $620 \mathrm{~K}$ on $\mathrm{Cu}(111)$ (Figure S5c and Figure S6c and d) and at $700 \mathrm{~K}$ on $\mathrm{Au}(111)$ (Figure S5d).

Notably, the benzo-fused double [7]helicene 2a that was obtained from precursor 1a in the solution synthesis ${ }^{47}$ was not observed at all in the on-surface experiments. Instead, the same precursor exclusively led to benzo-fused perihexacence $3 \mathrm{a}$. The rotation around the $\mathrm{C}-\mathrm{C}$ bond between the naphthyl group and the inner benzene unit of the $p$-terphenyl is relatively free in solution. Thus, bond formation seems possible at both the $\alpha$ - and the $\beta$-positions of the naphthyl moiety. Nevertheless, assuming that the oxidative cyclodehydrogenation of precursor 1a proceeds after the initial electron transfer, thus generating 
its radical cation of species, the formation of $2 \mathrm{a}$ in solution can be ascribed to the higher spin density at the $\alpha$-position according to the DFT calculation at the UB3LYP/6-31G(d,p) level (Figure S7). On the other hand, while not being perfectly flat, precursor 1a adopts a conformation that maximizes the contacts between the $\pi$-systems and the surface, as observed using STM (see Figure 2a, d, and g). Maintaining these contacts throughout the reaction will facilitate a flat product structure, namely, dibenzoperihexacene 3a. The adsorption energy of $1 \mathrm{a}$ on the $\mathrm{Cu}(110)$ surface was estimated to be $-2.48 \mathrm{eV}$ from the reactive force field (ReaxFF) simulation (see Table S1), which is consistent with the observed conformation. Furthermore, the on-surface cyclodehydrogenation supposedly proceeds via the initial abstraction of a hydrogen atom, ${ }^{25,28}$ thereby generating free radical species of the naphthyl group, followed by the $\mathrm{C}-\mathrm{C}$ bond formation with the central benzene ring. The reaction enthalpies and barriers for the hydrogen abstraction from naphthalene were calculated to be almost identical for the $\alpha$ - and $\beta$-positions, ${ }^{49,50}$ which can explain the observed difference in the region selectivity. Further, the rotation of the naphthyl groups on the surface is hindered by the higher rotation barriers of $0.44 \mathrm{eV}$ on $\mathrm{Au}(111)$ and $0.46 \mathrm{eV}$ on $\mathrm{Cu}(110)$ in comparison to that of $0.32 \mathrm{eV}$ in solution (see Table S1). It is worth noting that the adsorption of $3 \mathbf{a}$ is much more stable than that of $\mathbf{2 a - 1}$ with a difference of $-4.65 \mathrm{eV}$ (Table S2), which also favors the planarization reaction. We propose the reaction processes as shown in Figure S8. Nonetheless, comprehensive calculations of the energy barrier of each reaction step as well as stabilization energy of each possible intermediate need to be done in future work to obtain deeper insights into the reaction pathways.

We noticed that a nonnegligible amount of the dibenzoperihexacene molecules were randomly connected to each other after annealing at $640 \mathrm{~K}$ (Figure S2f), probably because the $t \mathrm{Bu}$ radicals were removed so that the cyclodehydrogenation was accompanied by intermolecular coupling. To suppress the oligomerization of the products, 2,2",6,6"-tetra(2-naphthyl)-pterphenyl (TNTP, 1b) was used as a precursor without $t \mathrm{Bu}$ groups for comparison. TNTP $\mathbf{1 b}$ was synthesized via a fourfold Suzuki reaction between 2,2",6,6"-tetrabromo$1,1^{\prime}: 4^{\prime}, 1^{\prime \prime}$-terphenyl ${ }^{47}$ and 2-naphthylboronic acid, as displayed in Scheme S1. TNTP $\mathbf{1 b}$ was deposited onto $\mathrm{Cu}(110)$, $\mathrm{Cu}(111)$, and $\mathrm{Au}(111)$ (Figure $\mathrm{S} 4 \mathrm{e}-\mathrm{h}$ ). When the same cyclodehydrogenation conditions as in the experiments with TNTP- $t \mathrm{Bu}$ 1a were applied, the TNTP molecules were planarized to dibenzoperihexacene $3 \mathbf{b}$ with much fewer intermolecular reactions (Figure $\mathrm{S} 5 \mathrm{a}-\mathrm{d}$ vs Figure $\mathrm{S} 5 \mathrm{e}-\mathrm{h}$ ). The yield of $\mathbf{3 b}$ on $\mathrm{Au}(111)$ was the highest compared to yields on other metal surfaces $(32 \%$ on $\mathrm{Cu}(110), 30 \%$ on $\mathrm{Cu}(111)$, and $56 \%$ on $\mathrm{Au}(111)$, as shown in Figure S5f-h). We thus took the reactions on $\mathrm{Au}(111)$ as a representative example.

After thermal sublimation, TNTP precursor $\mathbf{1 b}$ was initially adsorbed in a more distorted conformation on $\mathrm{Au}(111)$ surfaces and self-assembled into one-dimensional (1D) molecular chains. The chains were not perfectly periodic, thereby indicating the coexistence of different adsorption geometries. As illustrated in Figure $4 \mathrm{c}$ and d, precursor $\mathbf{1 b}$ was transformed into dibenzoperihexacene $3 \mathbf{b}$ after annealing at $720 \mathrm{~K}$ for $30 \mathrm{~min}$. Extending the annealing time leads to an increased yield of the planarization reaction. However, it also leads to an increase in intermolecular connections. Basing on trial-and-error experiments, we finally determined $30 \mathrm{~min}$ as
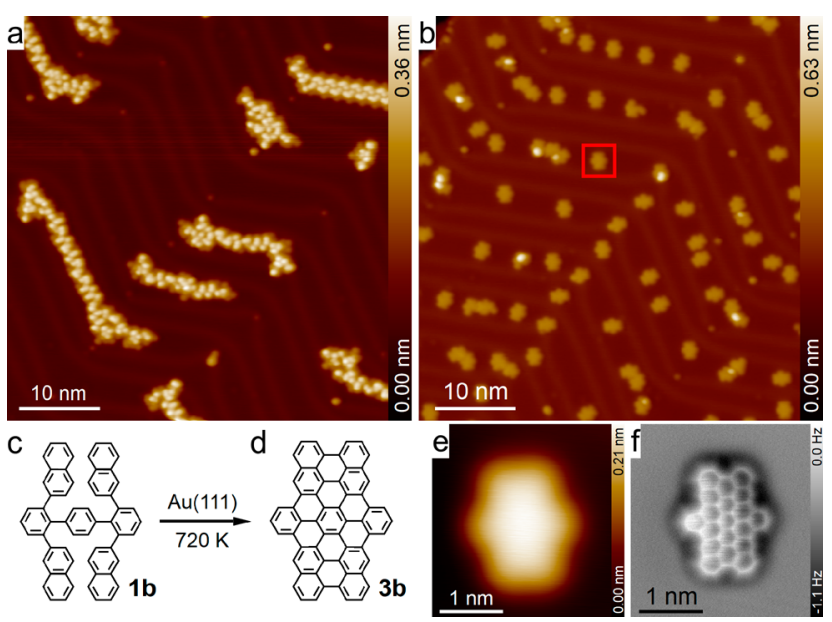

Figure 4. Cyclodehydrogenation reactions of 2,2",6,6"-tetra(2naphthyl)-p-terphenyl (TNTP, 1b) on $\mathrm{Au}(111)$. (a) STM overview of the self-assembly of TNTP $\mathbf{1 b}$ molecules on $\mathrm{Au}(111)$; (b) STM overview of the TNTP/Au(111) sample after annealing at $720 \mathrm{~K}$ for $30 \mathrm{~min}$. (c,d) Chemical structures of TNTP $\mathbf{1 b}$ and dibenzoperihexacene $3 \mathbf{b}$. (e) STM image of a single planarized product molecule marked with a red box in (b); (f) corresponding frequency shift AFM image. Imaging parameters: (a, b) $-1.0 \mathrm{~V}, 10 \mathrm{pA}$; (e) $1.0 \mathrm{~V}, 100 \mathrm{pA}$; and (d) tip height $Z=-50 \mathrm{pm}$, where the - sign means a decrease of the tip-sample distance relative to the STM set point $(0.1 \mathrm{~V}, 10 \mathrm{pA})$ on the adjacent $\mathrm{Au}(111)$ surfaces.

the optimal annealing time. The majority of the planarized product molecules remained separate from each other (Figure $4 \mathrm{~b}$ ), in contrast to the randomly connected products that were obtained from TNTP- $t \mathrm{Bu}$ 1a (see Figure S2f and Figure S5c and $d$ ). The exact chemical structure of dibenzoperihexacene $3 \mathrm{~b}$ was determined using bond-resolution AFM (Figure 4f).

Finally, we exploited the on-surface regioselective cyclodehydrogenation to synthesize an even higher homologue of dibenzoperiacene, namely, dibenzoperioctacene 6 (Figure $4 \mathrm{~b}$ ). To this end, 4,4"-di-tert-butyl-2,2",6,6"-tetra(2-anthryl)-pterphenyl (TATP-tBu, 5, Figure 5a) was designed as the precursor and synthesized via the fourfold Suzuki coupling

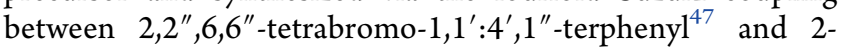
anthrylboronic acid, as described in Scheme S2. After sublimation at $720 \mathrm{~K}$ onto the $\mathrm{Au}(111)$ surface that was kept at room temperature, the TATP- $t \mathrm{Bu}$ molecules dominantly self-assembled into $1 \mathrm{D}$ molecular chains (Figure $5 c$ ). The STM image of TATP- $t$ Bu 5 (Figure $5 e$ ) indicated that three of the four anthryl groups lie more or less flat while the remaining one is highly tilted. Annealing the sample at 690 $\mathrm{K}$ for $30 \mathrm{~min}$ promoted the transformation from $\mathbf{5}$ to dibenzoperioctacene 6 , which was confirmed by the chemical bond imaging (Figure 5g).

The electronic structures of dibenzoperihexacenes $3 a$ and $3 b$ and dibenzoperioctacene 6 were characterized using scanning tunneling spectroscopy (STS) on the $\mathrm{Au}(111)$ surfaces. The STS curves in Figure 6a, e, and i recorded the $\mathrm{d} I / \mathrm{d} V$ versus bias voltage in the range from -2 to $2 \mathrm{~V}$ at specific points that were marked in the STM images in Figure $6 \mathrm{~b}, \mathrm{f}$, and $\mathrm{j}$, respectively. The characteristic peaks at -0.6 and $1.5 \mathrm{~V}$ in Figure $6 \mathrm{a}$ and $\mathrm{e}$ were assigned as the highest occupied molecular orbital (HOMO) and the lowest unoccupied molecular orbital (LUMO), respectively, for both $\mathbf{3 a}$ and $\mathbf{3 b}$. Therefore, a HOMO-LUMO energy gap of approximately 2.1 $\mathrm{eV}$ was deduced for dibenzoperihexacenes $\mathbf{3} \mathbf{a}$ and $\mathbf{3 b}$. Similarly, 


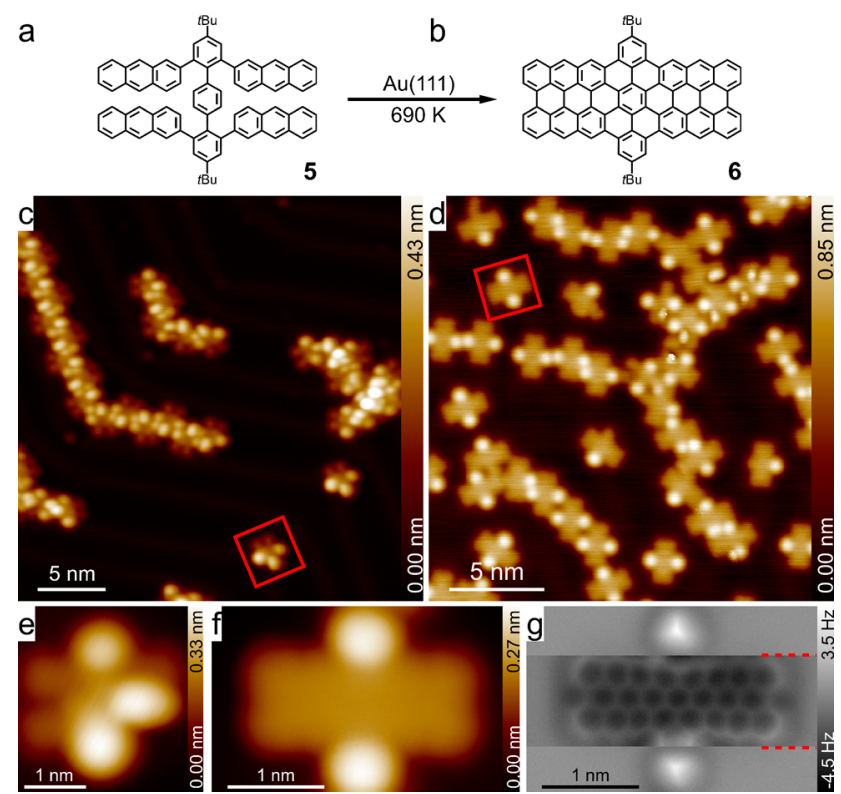

Figure 5. Synthesis of dibenzoperioctacene from TATP- $t \mathrm{Bu} 5$ on $\mathrm{Au}(111)$. (a, b) Chemical structures of TATP-tBu 5 and dibenzoperioctacene 6 (with $t \mathrm{Bu}$ groups). (c) STM overview of the self-assembled TATP-tBu 5 molecules on $\mathrm{Au}(111)$; (d) STM overview taken after annealing the sample at $690 \mathrm{~K}$ for $30 \mathrm{~min}$. (e) Zoomed-in STM image of an individual TATP- $t \mathrm{Bu} 5$ molecule that was marked with a red box in (c). (f) Zoomed-in STM image of a single dibenzoperioctacene 6 molecule that was marked with a red box in (d); (g) corresponding frequency shift AFM image. Imaging parameters: (c) $-1.0 \mathrm{~V}, 10 \mathrm{pA}$; (d,f) $0.1 \mathrm{~V}, 10 \mathrm{pA}$; (e) $-1.0 \mathrm{~V}, 100$ $\mathrm{pA}$; and $(\mathrm{g})$ tip height $Z=+170 \mathrm{pm},(Z=-20 \mathrm{pm}$ for the region between the two red dashed lines in $(\mathrm{g}))$, where the \pm signs represent the increase/decrease of the tip-sample distance relative to the STM set point $(0.1 \mathrm{~V}, 10 \mathrm{pA})$ on the adjacent $\mathrm{Au}(111)$ surfaces.
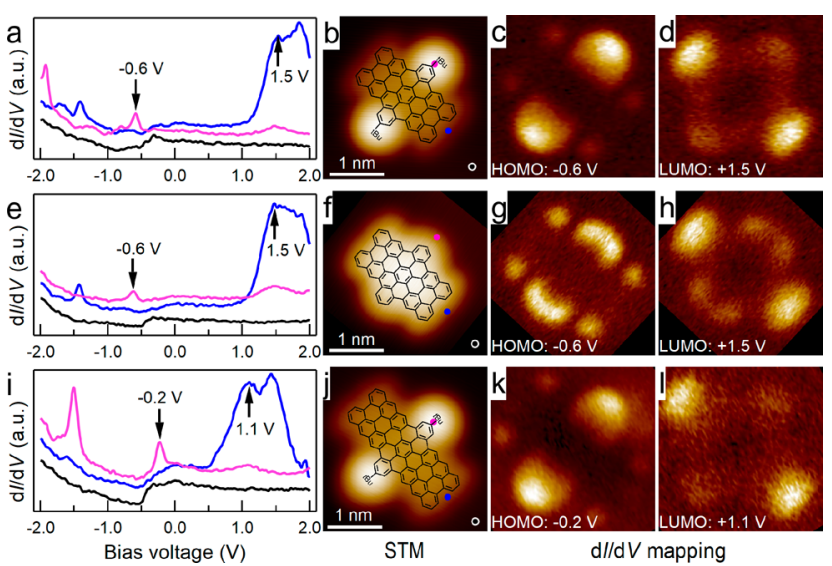

Figure 6. Electronic properties of dibenzoperihexacenes $\mathbf{3 a}$ and $\mathbf{3 b}$ (with and without $t \mathrm{Bu}$ groups) and dibenzoperioctacene 6 (with $t \mathrm{Bu}$ groups) measured on $\mathrm{Au}(111)$. (a, e, i) STS spectroscopies recorded at the positions marked in the corresponding STM images $(b, f, j)$ with the same colored dots. The spectra are vertically offset from each other for clarity. (b, f, j) STM images of the individual nanographene molecules with the corresponding molecular structures overlaid (imaging set point: $(b, f, j)-1.0 \mathrm{~V}, 100 \mathrm{pA}$; and $(\mathrm{c}, \mathrm{d}, \mathrm{g}, \mathrm{h}, \mathrm{k}, \mathrm{l})$ $\mathrm{d} I / \mathrm{d} V$ maps recorded at $-0.6 \mathrm{~V},+1.5 \mathrm{~V},-0.6 \mathrm{~V},+1.5 \mathrm{~V},-0.2 \mathrm{~V}$, and $+1.1 \mathrm{~V}$, respectively).

a HOMO-LUMO gap of $1.3 \mathrm{eV}$ was measured for dibenzoperioctacene 6 , thus highlighting the drastic decrease of the energy gap upon the structural extension and coinciding with the decreased HOMO-LUMO gaps of the larger periacene. ${ }^{51-53}$ Moreover, two-dimensional STS maps of the HOMO and LUMO for dibenzoperihexacenes 3a (Figure 5c and $\mathrm{d}$, respectively), $3 \mathbf{b}$ (Figure $5 \mathrm{~g}$ and $\mathrm{h}$, respectively), and dibenzoperioctacene 6 (Figure $5 \mathrm{k}$ and $\mathrm{l}$, respectively) were obtained at constant current. The spatially localized distribution of the electronic states indicated a weaker interference from the $\mathrm{Au}(111)$ surfaces compared to other more reactive surfaces, e.g., $\mathrm{Cu}(111)$ (see Figure S14), which enabled the detection of the molecular frontier orbitals. As seen in Figure $6 c, g$, and $k$, the HOMO of $3 a, 3 b$, and $\mathbf{6}$ mainly exists at the zigzag edges, which is reminiscent of the edge states observed in the zigzag-edge graphene nanoribbons. ${ }^{54}$ The HOMO localized at the zigzag edges is enhanced in the middle, which is where the zigzag edges are fused with a benzene ring. The similarity in the electronic structures of $\mathbf{3 a}$ and $\mathbf{3 b}$ indicates that the influence of $t \mathrm{Bu}$ groups on the molecular electronic properties is negligible.

\section{CONCLUSION}

In summary, we demonstrate that the regioselectivity of the cyclodehydrogenation of 2,2",6,6"-tetra(2-naphthyl)-p-terphenyl on metal surfaces is different from that in solution, thereby leading to an unprecedented synthesis of dibenzoperihexacene on $\mathrm{Cu}(110), \mathrm{Cu}(111)$, and $\mathrm{Au}(111)$ in contrast to the previously reported double [7] helicene generated through the solution synthesis. To the best of our knowledge, this is the first example to demonstrate different regioselectivity in similar reactions on the surface and in solution, thereby yielding different products from the same precursor. We have further developed a 2,2",6,6"-tetra(2-anthryl)-p-terphenyl precursor to accomplish the on-surface synthesis of dibenzoperioctacene as the largest periacene analogue with a small HOMO-LUMO gap of $1.3 \mathrm{eV}$. The current results provide new insight into the cyclodehydrogenation reactions on metal surfaces and a useful guideline for the design of new precursors, e.g., those having naphthyl and/or anthryl groups, for developing further varieties of nanographene structures. The structural extension to dibenzoperidecacene and the synthesis of benzo-fused zigzag graphene nanoribbons ${ }^{55}$ are planned for future investigation in our laboratories.

\section{METHODS}

Synthesis of Precursors. 4,4"-Di-tert-butyl-2,2",6,6"-tetra(2naphthyl)-p-terphenyl (TNTP-tBu, 1a) was synthesized as described in our previous report. ${ }^{47}$ The details of the synthesis of $2,2^{\prime \prime}, 6,6^{\prime \prime}$. tetra(2-naphthyl)-p-terphenyl (TNTP, 1b) and 4,4"-ditert-butyl2,2",6,6"-tetra(2-anthryl)-p-terphenyl (TATP-tBu, 5) are provided in Schemes S1 and S2, respectively.

Sample Preparation. The $\mathrm{Cu}(110) / \mathrm{Cu}(111) / \mathrm{Au}(111)$ crystals that were purchased from MaTeck (Germany) were cleaned using combined sputtering/annealing cycles. The precursors were deposited onto the single crystal surfaces from a homemade molecule evaporator. ${ }^{56}$ Reactions were triggered by heating the sample at appropriate temperatures for $30 \mathrm{~min}$. The prepared samples were transferred into an STM/AFM scanner where the base pressure is better than $1.0 \times 10^{-10}$ mbar.

STM/AFM and STS Measurements. All of the STM/AFM images were measured at $5 \mathrm{~K}$ with a commercial LT-STM/AFM (Scienta Omicron, Germany). The tip was grounded and a voltage bias was applied to the sample for STM imaging in the constant current mode. The STS spectra $(\mathrm{d} I / \mathrm{d} V$ vs bias voltage $(V))$ were acquired in the following steps: (i) Put the tip at the target positions with a tunneling set point of $-1 \mathrm{~V}$ and $100 \mathrm{pA}$, (ii) switch off the tipheight feedback, and (iii) ramp $V$ from -2 to $2 \mathrm{~V}$ and record the $\mathrm{dI} /$ 
$\mathrm{d} V$ signal vs $V$ using a lock-in amplifier $\left(V_{\mathrm{rms}}=20 \mathrm{mV}\right)$. The AFM images are constant-height frequency shift images that were acquired by using a tuning fork force sensor with $\mathrm{CO}$-functionalized tips and a frequency modulation mode. The resonance frequency of the force sensor is approximately $27 \mathrm{kHz}$. Vibration amplitudes of 51-68 pm were used for all the AFM images. The quality factors are $26.6 \mathrm{k}-$ $39.5 \mathrm{k}$.

Calculations. For the reactive force field (ReaxFF) simulations, we used LAMMPS (1 Feb 2014 version $)^{57}$ with the USER-REAXC package and fix qeq/reax. ${ }^{58}$ All the density functional theory (DFT) calculations were performed using the Vienna $\mathrm{Ab}$ initio Simulation Package (VASP), in which the electron-ion interactions were described using the Projector Augmented-Wave (PAW) potentials. $^{59,60}$ The exchange-correlation interactions were interpreted through the Perdew-Burke-Enzerhof (PBE) of the Generalized Gradient Approximation (GGA) pseudopotentials. ${ }^{61}$ The cutoff energy for the plane wave was set as $400 \mathrm{eV}$. The vdW-D3 method developed by Grimme et al. was employed to describe the van der Waals interactions. ${ }^{62}$ The $\Gamma$-center was adopted in all DFT calculations. ${ }^{63}$ The $\mathrm{Cu}(110)$ surface was modeled using periodic slabs consisting of four $\mathrm{Cu}$ atomic layers. A $15 \AA$ vacuum layer was included in order to avoid the interactions between the periodical cells. During the structural optimizations, the bottom two layers of $\mathrm{Cu}$ atoms were kept fixed, and all other atoms were relaxed until the atomic force was less than $0.01 \mathrm{eV} / \AA$. The simulations of the constant-height frequency shift AFM images were carried out using the probe particle model from Hapala et al. ${ }^{64,65}$ and taking the DFT calculated molecular structures as input. A CO tip was used with a lateral stiffness of $0.5 \mathrm{~N} \cdot \mathrm{m}^{-1}$ and an oscillation amplitude of $1 \AA$.

\section{ASSOCIATED CONTENT}

\section{S Supporting Information}

The Supporting Information is available free of charge on the ACS Publications website at DOI: 10.1021/jacs.9b01267.

AFM experimental images, DFT calculations on the adsorption geometries and AFM simulation images of the initial, intermediate and final state of TNTP-tBu 1a on $\mathrm{Cu}(110)$. Additional STM overviews of the reactions of $1 \mathrm{a}, 1 \mathrm{~b}$, and 5 on $\mathrm{Cu}(110), \mathrm{Cu}(111)$, and $\mathrm{Au}(111)$, respectively. Calculations on the adsorption energy of $1 \mathrm{a}$ and the rotation barrier of the naphthyl groups in solution and on $\mathrm{Au}(111)$ and $\mathrm{Cu}(110)$. Calculations on the adsorption energy of $\mathbf{3 a}$ and $\mathbf{2 a - 1}$ on $\mathrm{Cu}(110)$. Spin density of the radical cation of precursor $\mathbf{1 b}$. Proposed the reaction processes. Synthesis and characterization of precursors $\mathbf{1 b}$ and $\mathbf{5}$. STM $\mathrm{d} I / \mathrm{d} V$ spectroscopy of the planarized products $3 a, 3 b$, and 6 on $\mathrm{Cu}(111)$. Figures S1-S14. Schemes S1 and S2. Tables S1 and S2 (PDF)

\section{AUTHOR INFORMATION}

\section{Corresponding Authors}

*chilf@suda.edu.cn

*muellen@mpip-mainz.mpg.de

*narita@mpip-mainz.mpg.de

\section{ORCID}

Yunbin Hu: 0000-0001-5346-7059

Daniel Ebeling: 0000-0001-5829-170X

Akimitsu Narita: 0000-0002-3625-522X

Klaus Müllen: 0000-0001-6630-8786

Lifeng Chi: 0000-0003-3835-2776

\section{Present Address}

${ }^{\mathbb{I}}$ Physics Department E20, Technical University of Munich, James-Franck-Straße 1, D-85748 Garching, Germany

\section{Author Contributions}

$\boldsymbol{\Delta}_{\text {Q.Z. and Y.H. contributed equally. }}$

\section{Notes}

The authors declare no competing financial interest.

\section{ACKNOWLEDGMENTS}

This work was supported by the Ministry of Science and Technology of People's Republic of China (2017YFA0205002) and the National Natural Science Foundation of China (NSFC, Grant Nos. 21790053 and 51821002). We thank the Collaborative Innovation Center of Suzhou Nano Science \& Technology and 111 project. We are also thankful for the financial support from the Max Planck Society and the European Union's Horizon 2020 research and innovation program under GrapheneCore1 (No. 696656). We acknowledge financial support from DFG via GRK (Research Training Group) 2204 "Substitute Materials for Sustainable Energy Technologies", the DFG project EBE535/1 and the laboratory of Materials Research (LaMa) of JLU”

\section{REFERENCES}

(1) Wang, X. Y.; Narita, A.; Müllen, K. Precision Synthesis Versus Bulk-Scale Fabrication of Graphenes. Nat. Rev. Chem. 2017, 2, No. 0100

(2) Cai, J. M.; Ruffieux, P.; Jaafar, R.; Bieri, M.; Braun, T.; Blankenburg, S.; Muoth, M.; Seitsonen, A. P.; Saleh, M.; Feng, X. L.; Müllen, K.; Fasel, R. Atomically Precise Bottom-up Fabrication of Graphene Nanoribbons. Nature 2010, 466, 470-473.

(3) Ruffieux, P.; Cai, J.; Plumb, N. C.; Patthey, L.; Prezzi, D.; Ferretti, A.; Molinari, E.; Feng, X.; Müllen, K.; Pignedoli, C. A.; Fasel, R. Electronic Structure of Atomically Precise Graphene Nanoribbons. ACS Nano 2012, 6, 6930-6935.

(4) Metzger, R. M. Unimolecular Electronics. Chem. Rev. 2015, 115, $5056-5115$

(5) Han, W.; Kawakami, R. K.; Gmitra, M.; Fabian, J. Graphene Spintronics. Nat. Nanotechnol. 2014, 9, 794-807.

(6) Loh, K. P.; Tong, S. W.; Wu, J. S. Graphene and Graphene-Like Molecules: Prospects in Solar Cells. J. Am. Chem. Soc. 2016, 138, 1095-1102.

(7) Son, Y. W.; Cohen, M. L.; Louie, S. G. Energy Gaps in Graphene Nanoribbons. Phys. Rev. Lett. 2006, 97, 216803.

(8) Fujii, S.; Enoki, T. Nanographene and Graphene Edges: Electronic Structure and Nanofabrication. Acc. Chem. Res. 2013, 46, 2202-2210.

(9) Kawasumi, K.; Zhang, Q. Y.; Segawa, Y.; Scott, L. T.; Itami, K. A Grossly Warped Nanographene and the Consequences of Multiple Odd-Membered-Ring Defects. Nat. Chem. 2013, 5, 739-744.

(10) Cheung, K. Y.; Xu, X. M.; Miao, Q. Aromatic Saddles Containing Two Heptagons. J. Am. Chem. Soc. 2015, 137, 39103914.

(11) Matsumoto, A.; Suzuki, M.; Kuzuhara, D.; Hayashi, H.; Aratani, N.; Yamada, H. Tetrabenzoperipentacene: Stable Five-Electron Donating Ability and a Discrete Triple-Layered $\beta$-Graphite Form in the Solid State. Angew. Chem., Int. Ed. 2015, 54, 8175-8178.

(12) Dorel, R.; Manzano, C.; Grisolia, M.; Soe, W. H.; Joachim, C.; Echavarren, A. M. Tetrabenzocircumpyrene: A Nanographene Fragment with an Embedded Peripentacene Core. Chem. Commun. 2015, 51, 6932-6935.

(13) Dumslaff, T.; Yang, B.; Maghsoumi, A.; Velpula, G.; Mali, K. S.; Castiglioni, C.; De Feyter, S.; Tommasini, M.; Narita, A.; Feng, X. L.; Müllen, K. Adding Four Extra K-Regions to Hexa-peri-hexabenzocoronene. J. Am. Chem. Soc. 2016, 138, 4726-4729.

(14) Beser, U.; Kastler, M.; Maghsoumi, A.; Wagner, M.; Castiglioni, C.; Tommasini, M.; Narita, A.; Feng, X. L.; Müllen, K. A C216Nanographene Molecule with Defined Cavity as Extended Coronoid. J. Am. Chem. Soc. 2016, 138, 4322-4325. 
(15) Simpson, C. D.; Brand, J. D.; Berresheim, A. J.; Przybilla, L.; Räder, H. J.; Müllen, K. Synthesis of a Giant 222 Carbon Graphite Sheet. Chem. - Eur. J. 2002, 8, 1424-1429.

(16) Tomović, Ž.; Watson, M. D.; Müllen, K. Superphenalene-Based Columnar Liquid Crystals. Angew. Chem., Int. Ed. 2004, 43, 755-758.

(17) Böhme, T.; Simpson, C. D.; Müllen, K.; Rabe, J. P. CurrentVoltage Characteristics of a Homologous Series of Polycyclic Aromatic Hydrocarbons. Chem. - Eur. J. 2007, 13, 7349-7357.

(18) Feng, X. L.; Liu, M. Y.; Pisula, W.; Takase, M.; Li, J. L.; Müllen, K. Supramolecular Organization and Photovoltaics of TriangleShaped Discotic Graphenes with Swallow-Tailed Alkyl Substituents. Adv. Mater. 2008, 20, 2684-2689.

(19) Narita, A.; Wang, X. Y.; Feng, X. L.; Müllen, K. New Advances in Nanographene Chemistry. Chem. Soc. Rev. 2015, 44, 6616-6643.

(20) Liu, J. Z.; Narita, A.; Osella, S.; Zhang, W.; Schollmeyer, D.; Beljonne, D.; Feng, X. L.; Müllen, K. Unexpected Scholl Reaction of 6,7,13,14-Tetraarylbenzo[k]tetraphene: Selective Formation of FiveMembered Rings in Polycyclic Aromatic Hydrocarbons. J. Am. Chem. Soc. 2016, 138, 2602-2608.

(21) Dou, X.; Yang, X. Y.; Bodwell, G. J.; Wagner, M.; Enkelmann, V.; Müllen, K. Unexpected Phenyl Group Rearrangement during an Intramolecular Scholl Reaction Leading to an Alkoxy-Substituted Hexa-peri-hexabenzocoronene. Org. Lett. 2007, 9, 2485-2488.

(22) Pradhan, A.; Dechambenoit, P.; Bock, H.; Durola, F. Twisted Polycyclic Arenes by Intramolecular Scholl Reactions of C3Symmetric Precursors. J. Org. Chem. 2013, 78, 2266-2274.

(23) Dötz, F.; Brand, J. D.; Ito, S.; Gherghel, L.; Müllen, K. Synthesis of Large Polycyclic Aromatic Hydrocarbons: Variation of Size and Periphery. J. Am. Chem. Soc. 2000, 122, 7707-7717.

(24) Müller, M.; Mauermann-Düll, H.; Wagner, M.; Enkelmann, V.; Müllen, K. A Cycloaddition Cyclodehydrogenation Route from Stilbenoids to Extended Aromatic-Hydrocarbons. Angew. Chem., Int. Ed. Engl. 1995, 34, 1583-1586.

(25) Treier, M.; Pignedoli, C. A.; Laino, T.; Rieger, R.; Müllen, K.; Passerone, D.; Fasel, R. Surface-Assisted Cyclodehydrogenation Provides a Synthetic Route towards Easily Processable and Chemically Tailored Nanographenes. Nat. Chem. 2011, 3, 61-67.

(26) Hieulle, J.; Carbonell-Sanromà, E.; Vilas-Varela, M.; GarciaLekue, A.; Guitián, E.; Peña, D.; Pascual, J. I. On-Surface Route for Producing Planar Nanographenes with Azulene Moieties. Nano Lett. 2018, 18, 418-423.

(27) Pinardi, A. L.; Martínez, J. I.; Jančař́k, A.; Stará, I. G.; Starý, I.; López, M. F.; Méndez, J.; Martin-Gago, J. Á. Sequential Formation of $\mathrm{N}$-doped Nanohelicenes, Nanographenes and Nanodomes by SurfaceAssisted Chemical (Cyclo)Dehydrogenation of Heteroaromatics. Chem. Commun. 2014, 50, 1555-1557.

(28) Björk, J. Reaction Mechanisms for On-Surface Synthesis of Covalent Nanostructures. J. Phys.: Condens. Matter 2016, 28, No. 083002 .

(29) Krüger, J.; García, F.; Eisenhut, F.; Skidin, D.; Alonso, J. M.; Guitián, E.; Pérez, D.; Cuniberti, G.; Moresco, F.; Peña, D. Decacene: On-Surface Generation. Angew. Chem., Int. Ed. 2017, 56, 1194511948 .

(30) Zuzak, R.; Dorel, R.; Kolmer, M.; Szymonski, M.; Godlewski, S.; Echavarren, A. M. Higher Acenes by On-Surface Dehydrogenation: from Heptacene to Undecacene. Angew. Chem., Int. Ed. 2018, 57, 10500-10505.

(31) Wang, X. Y.; Richter, M.; He, Y. Q.; Björk, J.; Riss, A.; Rajesh, R.; Garnica, M.; Hennersdorf, F.; Weigand, J. J.; Narita, A.; Berger, R.; Feng, X. L.; Auwärter, W.; Barth, J. V.; Palma, C. A.; Müllen, K. Exploration of Pyrazine-Embedded Antiaromatic Polycyclic Hydrocarbons Generated by Solution and On-Surface Azomethine Ylide Homocoupling. Nat. Commun. 2017, 8, 1948.

(32) Zuzak, R.; Castro-Esteban, J.; Brandimarte, P.; Engelund, M.; Cobas, A.; Piątkowski, P.; Kolmer, M.; Pérez, D.; Guitián, E.; Szymonski, M.; Sánchez-Portal, D.; Godlewski, S.; Peña, D. Building a 22-Ring Nanographene by Combining In-Solution and On-Surface Syntheses. Chem. Commun. 2018, 54, 10256-10259.
(33) Rogers, C.; Chen, C.; Pedramrazi, Z.; Omrani, A. A.; Tsai, H. Z.; Jung, H. S.; Lin, S.; Crommie, M. E.; Fischer, F. R. Closing the Nanographene Gap: Surface-Assisted Synthesis of Peripentacene from 6,6'-Bipentacene Precursors. Angew. Chem., Int. Ed. 2015, 54, 1514315146.

(34) Pavliček, N.; Mistry, A.; Majzik, Z.; Moll, N.; Meyer, G.; Fox, D. J.; Gross, L. Synthesis and Characterization of Triangulene. Nat. Nanotechnol. 2017, 12, 308-311.

(35) Sun, Z.; Zeng, Z. B.; Wu, J. S. Zethrenes, Extended pQuinodimethanes, and Periacenes with a Singlet Biradical Ground State. Acc. Chem. Res. 2014, 47, 2582-2591.

(36) Sun, Z.; Ye, Q.; Chi, C. Y.; Wu, J. S. Low Band Gap Polycyclic Hydrocarbons: from Closed-Shell Near Infrared Dyes and Semiconductors to Open-Shell Radicals. Chem. Soc. Rev. 2012, 41, 78577889.

(37) Li, J. L.; Zhang, K.; Zhang, X. J.; Huang, K. W.; Chi, C. Y.; Wu, J. S. meso-Substituted Bisanthenes as Soluble and Stable Near-Infrared Dyes. J. Org. Chem. 2010, 75, 856-863.

(38) Zhang, K.; Huang, K. W.; Li, J. L.; Luo, J.; Chi, C. Y.; Wu, J. S. A Soluble and Stable Quinoidal Bisanthene with NIR Absorption and Amphoteric Redox Behavior. Org. Lett. 2009, 11, 4854-4857.

(39) Fort, E. H.; Scott, L. T. One-Step Conversion of Aromatic Hydrocarbon Bay Regions into Unsubstituted Benzene Rings: a Reagent for the Low-Temperature, Metal-Free Growth of SingleChirality Carbon Nanotubes. Angew. Chem., Int. Ed. 2010, 49, 66266628.

(40) Fort, E. H.; Donovan, P. M.; Scott, L. T. Diels-Alder Reactivity of Polycyclic Aromatic Hydrocarbon Bay Regions: Implications for Metal-Free Growth of Single-Chirality Carbon Nanotubes. J. Am. Chem. Soc. 2009, 131, 16006-16007.

(41) Clar, E. Synthesis of Ovalene. Nature 1948, 161, 238-239.

(42) Würthner, F. Perylene Bisimide Dyes as Versatile Building Blocks for Functional Supramolecular Architectures. Chem. Commun. 2004, 1564-1579.

(43) Akamatu, H.; Inokuchi, H.; Matsunaga, Y. Electrical Conductivity of the Perylene-Bromine Complex. Nature 1954, 173, $168-169$.

(44) Ni, Y.; Gopalakrishna, T. Y.; Phan, H.; Herng, T. S.; Wu, S. F.; Han, Y.; Ding, J.; Wu, J. S. A peri-Tetracene Diradicaloid: Synthesis and Properties. Angew. Chem., Int. Ed. 2018, 57, 9697-9701.

(45) Ajayakumar, M. R.; Fu, Y. B.; Ma, J.; Hennersdorf, F.; Komber, H.; Weigand, J. J.; Alfonsov, A.; Popov, A. A.; Berger, R.; Liu, J. Z.; Müllen, K.; Feng, X. L. Toward Full Zigzag-Edged Nanographenes: peri-Tetracene and its Corresponding Circumanthracene. J. Am. Chem. Soc. 2018, 140, 6240-6244.

(46) Wang, X. Y.; Dienel, T.; Di Giovannantonio, M.; Barin, G. B.; Kharche, N.; Deniz, O.; Urgel, J. I.; Widmer, R.; Stolz, S.; De Lima, L. H.; Muntwiler, M.; Tommasini, M.; Meunier, V.; Ruffieux, P.; Feng, X. L.; Fasel, R.; Müllen, K.; Narita, A. Heteroatom-Doped Perihexacene from a Double Helicene Precursor: On-Surface Synthesis and Properties. J. Am. Chem. Soc. 2017, 139, 4671-4674.

(47) Hu, Y. B.; Wang, X. Y.; Peng, P. X.; Wang, X. C.; Cao, X. Y.; Feng, X. L.; Müllen, K.; Narita, A. Benzo-Fused Double [7]Carbohelicene: Synthesis, Structures, and Physicochemical Properties. Angew. Chem., Int. Ed. 2017, 56, 3374-3378.

(48) Gross, L.; Mohn, F.; Moll, N.; Liljeroth, P.; Meyer, G. The Chemical Structure of a Molecule Resolved by Atomic Force Microscopy. Science 2009, 325, 1110-1114.

(49) Hemelsoet, K.; Van Speybroeck, V.; Moran, D.; Marin, G. B.; Radom, L.; Waroquier, M. Thermochemistry and Kinetics of Hydrogen Abstraction by Methyl Radical from Polycyclic Aromatic Hydrocarbons. J. Phys. Chem. A 2006, 110, 13624-13631.

(50) Carissan, Y.; Klopper, W. Hydrogen Abstraction from Biphenyl, Acenaphthylene, Naphthalene and Phenanthrene by Atomic Hydrogen and Methyl Radical: DFT and G3(MP2)-RAD Data. J. Mol. Struct:: THEOCHEM 2010, 940, 115-118.

(51) Plasser, F.; Pašalić, H.; Gerzabek, M. H.; Libisch, F.; Reiter, R.; Burgdörfer, J.; Müller, T.; Shepard, R.; Lischka, H. The Multiradical 
Character of One- and Two-Dimensional Graphene Nanoribbons. Angew. Chem., Int. Ed. 2013, 52, 2581-2584.

(52) Hod, O.; Barone, V.; Scuseria, G. E. Half-Metallic Graphene Nanodots: A Comprehensive First-Principles Theoretical Study. Phys. Rev. B: Condens. Matter Mater. Phys. 2008, 77, No. 035411.

(53) Rivero, P.; Jiménez-Hoyos, C. A.; Scuseria, G. E. Entanglement and Polyradical Character of Polycyclic Aromatic Hydrocarbons Predicted by Projected Hartree-Fock Theory. J. Phys. Chem. B 2013, 117, 12750-12758.

(54) Ruffieux, P.; Wang, S. Y.; Yang, B.; Sánchez-Sánchez, C.; Liu, J.; Dienel, T.; Talirz, L.; Shinde, P.; Pignedoli, C. A.; Passerone, D.; Dumslaff, T.; Feng, X. L.; Müllen, K.; Fasel, R. On-Surface Synthesis of Graphene Nanoribbons with Zigzag Edge Topology. Nature 2016, 531, 489-493.

(55) Chen, L. P.; Wang, L. J.; Beljonne, D. Designing Coved Graphene Nanoribbons with Charge Carrier Mobility Approaching That of Graphene. Carbon 2014, 77, 868-879.

(56) Zint, S.; Ebeling, D.; Ahles, S.; Wegner, H. A.; Schirmeisen, A. Subsurface-Controlled Angular Rotation: Triphenylene Molecules on $\mathrm{Au}(111)$ Substrates. J. Phys. Chem. C 2016, 120, 1615-1622.

(57) Plimpton, S. Fast Parallel Algorithms for Short-Range Molecular Dynamics. J. Comput. Phys. 1995, 117, 1-19.

(58) Aktulga, H. M.; Fogarty, J. C.; Pandit, S. A.; Grama, A. Y. Parallel Reactive Molecular Dynamics: Numerical Methods and Algorithmic Techniques. Parallel Comput. 2012, 38, 245-259.

(59) Kresse, G.; Furthmüller, J. Efficient Iterative Schemes for $a b$ initio Total-Energy Calculations Using a Plane-Wave Basis Set. Phys. Rev. B: Condens. Matter Mater. Phys. 1996, 54, 11169.

(60) Blöchl, P. E. Projector Augmented-Wave Method. Phys. Rev. B: Condens. Matter Mater. Phys. 1994, 50, 17953.

(61) Perdew, J. P.; Burke, K.; Ernzerhof, M. Generalized Gradient Approximation Made Simple. Phys. Rev. Lett. 1996, 77, 3865.

(62) Grimme, S.; Antony, J.; Ehrlich, S.; Krieg, H. A. A Consistent and Accurate $a b$ initio Parametrization of Density Functional Dispersion Correction (DFT-D) for the 94 Elements H-Pu. J. Chem. Phys. 2010, 132, 154104.

(63) Monkhorst, H. J.; Pack, J. D. Special Points for Brillouin-Zone Integrations. Phys. Rev. B 1976, 13, 5188.

(64) Hapala, P.; Kichin, G.; Wagner, C.; Tautz, F. S.; Temirov, R.; Jelínek, P. Mechanism of High-Resolution STM/AFM Imaging with Functionalized Tips. Phys. Rev. B: Condens. Matter Mater. Phys. 2014, 90, No. 085421.

(65) Hapala, P.; Temirov, R.; Tautz, F. S.; Jelínek, P. Origin of HighResolution IETS-STM Images of Organic Molecules with Functionalized Tips. Phys. Rev. Lett. 2014, 113, 226101. 